\title{
ALTERED APOPTOTIC PROFILES IN IRRADIATED PATIENTS WITH INCREASED TOXICITY
}

\author{
Nigel E. A. Crompton, Ph.D., D.Sc., * Raymond Miralbell, M.D., ${ }^{\dagger}$ Hans-Peter Rutz, M.D., \\ Fügen Ersoy, M.D., ${ }^{\S}$ Özden Sanal, M.D., ${ }^{\S}$ Danielle Wellmann, M.D.," Sabine Bieri, M.D., ${ }^{\mathrm{q}}$ \\ Philippe A. Coucke, M.D.," Gillian C. Emery, B.Sc.,* Yu-Quan Shi, M.D.,** \\ Hans Blattmann, Ph.D., * and Mahmut Ozsahin, M.D., Ph.D."
}

*Radiation Medicine, Life Sciences Department, Paul Scherrer Institute (PSI), Switzerland; †Department of Radiation Oncology, Hôpital Cantonal Universitaire de Genève (HCUG), Geneva, Switzerland; ${ }^{*}$ Department of Radiation Oncology, Kantonsspital Winterthur, Switzerland; 'Immunology Division, Hacettepe Children's Hospital, Ankara, Turkey; "Department of Radiation Oncology,

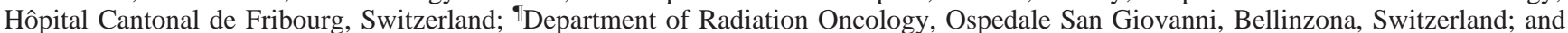
"Department of Radiation Oncology, Centre Hospitalier Universitaire Vaudois (CHUV), Switzerland

\begin{abstract}
Purpose: A retrospective study of radiation-induced apoptosis in CD4 and CD8 T-lymphocytes, from 12 cancer $\overline{\text { patients }}$ who displayed enhanced toxicity to radiation therapy and 9 ataxia telangiectasia patients, was performed to test for altered response compared to healthy blood-donors and normal cancer patients.

Methods and Materials: Three milliliters of heparinized blood from each donor was sent via express post to the Paul Scherrer Institute (PSI) for subsequent examination. The blood was diluted 1:10 in RPMI medium, irradiated with 0-, 2-, or 9-Gy X-rays, and incubated for 48 h. CD4 and CD8 T-lymphocytes were then labeled using FITC-conjugated antibodies, erythrocytes were lysed, and the DNA stained with propidium iodide. Subsequently, cells were analyzed using a Becton Dickinson FACScan flow cytometer. Radiation-induced apoptosis was recognized in leukocytes as reduced DNA content attributed to apoptosis-associated changes in chromatin structure. Apoptosis was confirmed by light microscopy, electron microscopy, and by the use of commercially available apoptosis detection kits (in situ nick translation and Annexin V). Data from hypersensitive individuals were compared to a standard database of 105 healthy blood-donors, and a database of 48 cancer patient blood donors who displayed normal toxicity to radiation therapy. To integrate radiosensitivity results from CD4 and CD8 T-lymphocytes after 2 and $9 \mathrm{~Gy}, z$-score analyses were performed.

Results: A cohort of 12 hypersensitive patients was evaluated; 8 showed enhanced early toxicity, 3 showed

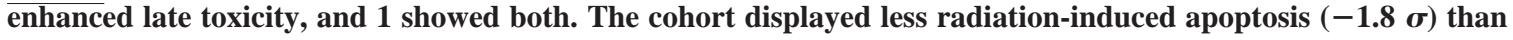
average age-matched donors. A cohort of 9 ataxia telangiectasia homozygotes displayed even less apoptosis $(-3.6$ $\boldsymbol{\sigma})$.

Conclusion: The leukocyte apoptosis assay appears to be a useful predictor of individuals likely to display increased toxicity to radiation therapy; however, validation of this requires a prospective study. (c) 1999 Elsevier Science Inc.
\end{abstract}

Normal tissue toxicity, Radiation-induced apoptosis, Ataxia telangiectasia, Concomitant chemotherapy, Lymphocytes.

\section{INTRODUCTION}

Development of predictive assays for clinical prediction requires that the diagnostic test employed display both high reproducibility and low variation (1). With the development of the leukocyte apoptosis assay to identify individuals genetically predisposed to radiation injury, both of these parameters were carefully considered. We have developed an assay employing a triple variation-minimizing strategy; flow cytometric blood analysis, gentle fixation, and cumu- lative apoptosis. By employing flow cytometric blood analysis 10,000 cells or more can be rapidly and objectively assessed for the parameter of interest (2). More importantly, a number of different cell types can be investigated, which enhances the specificity of the assay, giving the clinician additional confidence in results from samples which deviate from normality (3). Because apoptosis is the biological endpoint measured, use of a gentle fixation procedure ensures that the fragile apoptotic cells are not being lost during the work-up. Although the process of apoptosis in many cell
Reprint requests to: Nigel E. A. Crompton, Ph.D., D.Sc., Radiation Medicine, Paul Scherrer Institute, CH-5232 Villigen-PSI, Switzerland. Tel: +41 (56) 31028 71; Fax: +41 (56) 31044 17; E-mail: crompton@psi.ch

Presented at the 40th Annual Meeting of the American Society for Therapeutic Radiology and Oncology, October 25-29, 1998, Phoenix, AZ. This was a multi-center collaboration of the Scientific Association of Swiss Radiation Oncology (SASRO).

Accepted for publication 28 April 1999. 
types is short-lived, lymphocytes in cell culture display an "arrested" apoptosis after radiation exposure. The cells remain in this state for many days, and accumulation of the apoptotic cells leads to large signal-to-noise ratios. We have also developed an assay employing a triple-enhanced reproducibility strategy; robust handling, standardized components, and a simple, optimized procedure. The assay is robust to handling. Subsequent to venipuncture, the peripheral blood lymphocytes can be left at room temperature for $72 \mathrm{~h}$ and can be readily transported in the mail. The components used are all commercially available and are all standard materials used for blood handling or flow cytometry. In addition, the assay is a relatively straightforward assay that is not burdened by critical procedures.

We have previously demonstrated that the population of dead cells evaluated was apoptotic $(4,5)$. The cells display a small size with a reduced propidium iodide signal. This has been previously reported in the literature, and is typical of apoptotic cells in general $(6-8)$. The cells are whole, but their membranes are permeable. Using light and electron microscopy methods, an ultrastructure typical for apoptotic cells, as described in the literature, is observed $(9,10)$. The nuclei contain condensed chromatin, and large vacuoles are visible in the cytoplasm (11). Biochemical analyses reveal the cells to be both TUNEL- and ANNEXIN V-positive (5). Further indications that what we were measuring were truly apoptotic cells have also been described in the literature, and include: (a) that this was by far the major form of radiation-induced cytotoxicity observed after radiation (1214), that lymphocytes from patients displaying ataxia telangiectasia display reduced yields of apoptosis (15-17), and that lymphocytes from umbilical cord blood display enhanced yields of apoptosis (18).

The various leukocyte cell types display clear differences in radiosensitivity $(4,19)$. Of the cell types investigated, monocytes were the most radioresistant, displaying little difference to background noise. The assay has been used to monitor apoptosis in granulocytes, and we have used it to monitor the health of granulocytes in novel bone marrow transplantation protocols (20). Of the lymphocyte cell types examined, CD8 T-lymphocytes appear the most sensitive; however, some individuals tested displayed similar CD8 and CD4 T-lymphocyte apoptosis yields (4). Differences in yields between the lymphocyte cell types are heavily dependent on the genotype of the individual, as we have demonstrated for patients with ataxia telangiectasia and immunodeficiency, centromeric instability, and facial anomalies (ICF) syndrome. Gender differences appeared to have no effect on the apoptotic yield; however, donor age did make a difference. Although originally described after $24 \mathrm{~h}$ post-incubation, little statistical significance could be attached to these early studies (5). With subsequent optimization of the assay, the analyses were performed $48 \mathrm{~h}$ after irradiation, and we report here a highly significant dependence on age of apoptotic yield. In this double-blind crosssectional study, we investigated radiation-induced apoptosis in T-lymphocytes from various cohorts of blood donors: healthy donors, normal cancer patients, cancer patients hypersensitive to radiotherapy, and ataxia telangiectasia homozygotes who are highly sensitive to ionizing radiation (15).

\section{METHODS AND MATERIALS}

Blood samples were collected from various sources. All blood samples were collected under consent and were transported to the Paul Scherrer Institute in heparin tubes at room temperature using conventional postal options. Usually, blood was examined within $48 \mathrm{~h}$ of venipuncture; rarely, it was examined $72 \mathrm{~h}$ later. The Swiss Red Cross provided blood samples from 105 healthy donors (average age 47.2 years, range 21-73). For the cancer patient cohort, 48 samples were received from a single clinic in Switzerland (average age 71.9 years, range 30-89). To collect a cohort of hypersensitive patients, request for assistance was made to the various radiation oncology departments in Switzerland. A total of 12 samples were accrued via four major centers. The average age of the patients was 55 years, with a range of 41-75. A standardized, confidential questionnaire was completed by the referring physician, which provided pertinent data concerning patient diagnosis, hypersensitive reaction, radiotherapy, concomitant chemotherapy, and probe accrual for each of the hypersensitive patients. For logistic reasons, blood from 2 hypersensitive patients (case numbers 3 and 8) were examined within $72 \mathrm{~h}$ of venipuncture. Blood samples from 9 ataxia telangiectasia homozygotes (average age 7.6 years, range 4-11.5), were provided from the Immunology Division, Hacettepe Children's Hospital, Ankara, Turkey. These samples were transported by air and were all examined within $48 \mathrm{~h}$ of venipuncture.

Details of the leukocyte apoptosis assay have been previously described (21). Briefly, heparinized whole blood (3.6 ml) was diluted to a 1:10 concentration in RPMI 1640 medium (Life Technologies, Basel, Switzerland) containing $20 \%$ FBS, and the cells were treated with 0-, 2-, and 9-Gy X-rays (Philips MCN 321 x-ray tube, Hamburg, Germany; $300 \mathrm{kVp}, 10.65 \mathrm{~mA}, 3.4 \mathrm{~Gy} / \mathrm{min})$. Dosimetry was performed using a Farmer dosimeter 2570 (Nuclear Enterprises, Zurzach, Switzerland; exact doses measured were 2.34 and $9.01 \mathrm{~Gy}$ ). After $48 \mathrm{~h}$, cells were harvested and treated for $20 \mathrm{~min}$ with either CD4 or CD8 FITC-conjugated monoclonal antibodies (Becton Dickinson, Basel, Switzerland); $10 \mathrm{ml}$ antibody suspension were added to 200-ml cell suspension. To promote lysis of the erythrocytes and permeabilization of leukocytes, the cell suspensions were incubated for $10 \mathrm{~min}$ at room temperature in a 4:1 diethyleneglycol:formaldehyde buffered solution (FACS Lysing Solution, Becton Dickinson, Basel, Switzerland). The cells were then washed, centrifuged, and resuspended in $200 \mathrm{ml}$ phosphate buffer (FACS Flow, Becton Dickinson) with 5 $\mathrm{ml}$ of $1 \mathrm{mg} / \mathrm{ml} \mathrm{PI}$ and $50 \mathrm{ml}$ of $1 \mathrm{mg} / \mathrm{ml} \mathrm{RNase} \mathrm{(Bovine}$ pancreas RNase A, Serva, Heidelberg, Germany). The cells were subsequently examined in the flow cytometer. 


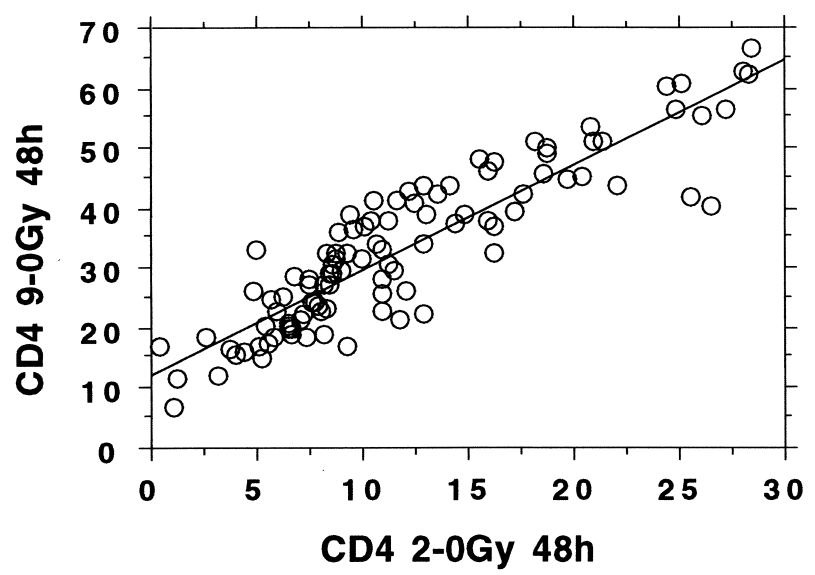

Fig. 1. Interdose comparison of the percent apoptosis induced by 2- and 9-Gy X-rays in CD4 T-lymphocytes from a cohort of 105 healthy Red Cross blood donors. The background levels (0 Gy) of apoptosis were subtracted.

To detect treatment-induced apoptosis, multiparameter data acquisition of the cell sample was performed using a FACScan flow cytometer (Becton Dickinson, San Jose, CA) with a $488 \mathrm{~nm}, 15 \mathrm{~mW}$ argon-ion laser (Coherent, Santa Clara, CA). Data from each lymphocyte sample were acquired immediately after the staining procedure. A twodimensional dot-plot of antibody-fluorescence (FL1-height) versus cellular DNA content (FL2-height) was used to identify the CD4- or CD8-positive lymphocytes. A two-dimensional dot-plot of cellular DNA content (FL2-height) versus cell size (FSC-height) was used to determine the proportion of apoptotic cells by identifying the population of cells with reduced DNA content and slightly reduced cell size. The two populations could always be distinguished. The apoptotic cells had a significantly reduced (approximately 5-fold, compared to non-apoptotic cells) DNA fluorescence. This 5-fold reduction remained constant over many days, and is attributed to chromatin condensation associated with the process of apoptosis (21). Radiation-induced apoptosis was calculated by subtracting the background level (0 Gy) of apoptotic cells from the total measured. Data are presented in this form, or age-weighted (by subtracting the expected apoptotic yield based on the regression curve of age versus apoptosis), or age-weighted as $z$-scores (standard deviation, $\sigma$, units from the mean).

\section{RESULTS}

To have a baseline with which to compare radiationinduced apoptosis in T-lymphocytes from patients displaying enhanced toxicity to radiation therapy, it was necessary to screen at least 100 healthy blood donors, using the leukocyte apoptosis assay. Interdose comparisons of the percent apoptosis induced by 2- and 9-Gy X-rays in CD4 T-lymphocytes from a cohort of 105 healthy Red Cross blood donors (average age 47.2 years; 29 females, 76 males) are displayed in Fig. 1. A strong correlation was observed

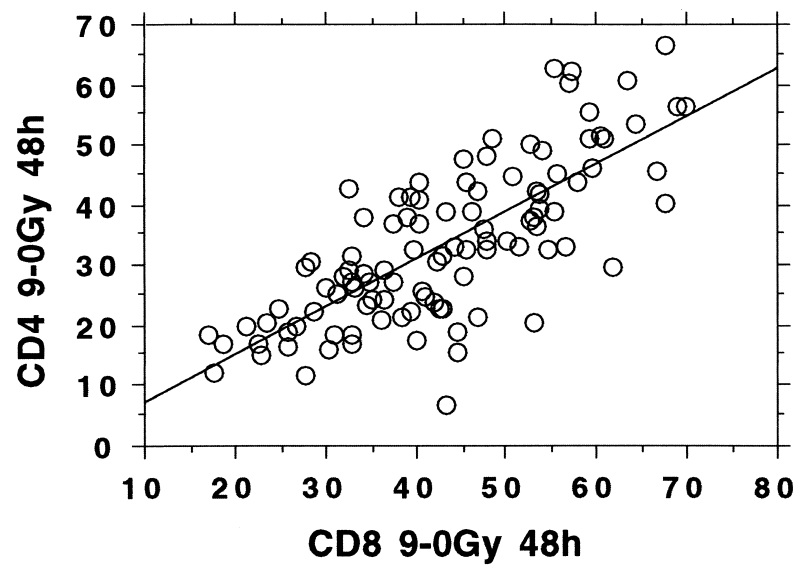

Fig. 2. Interleukocyte comparison of the percent apoptosis induced by 9-Gy X-rays in CD4 and CD8 T-lymphocytes from a cohort of 105 healthy Red Cross blood donors. The background levels (0 Gy) of apoptosis were subtracted.

$(r=0.9)$ for CD4 T-lymphocytes. The data are presented with the background (0 Gy) apoptosis levels subtracted. A similar analysis of the CD8 T-lymphocytes resulted in $r=$ 0.74. The background levels of apoptosis for the two cell types averaged $4 \%$ and $4.8 \%$ for the CD4 and CD8 Tlymphocytes, respectively. No correlation between background apoptosis and radiation-induced apoptosis was observed, as previously reported (5). The levels of apoptosis observed after 9-Gy X-rays in the CD4 and CD8 T-lymphocytes are compared in Fig. 2. The regression line has the formula $\mathrm{y}=0.79 \mathrm{x}-0.39, r=0.77$. The correlation between radiation-induced apoptosis in CD4 T-lymphocytes and age of donor is displayed in Fig. 3. Included are two cohorts of donors; the 105 Red Cross donors and 48 cancer patient donors whose average age was almost 20 years

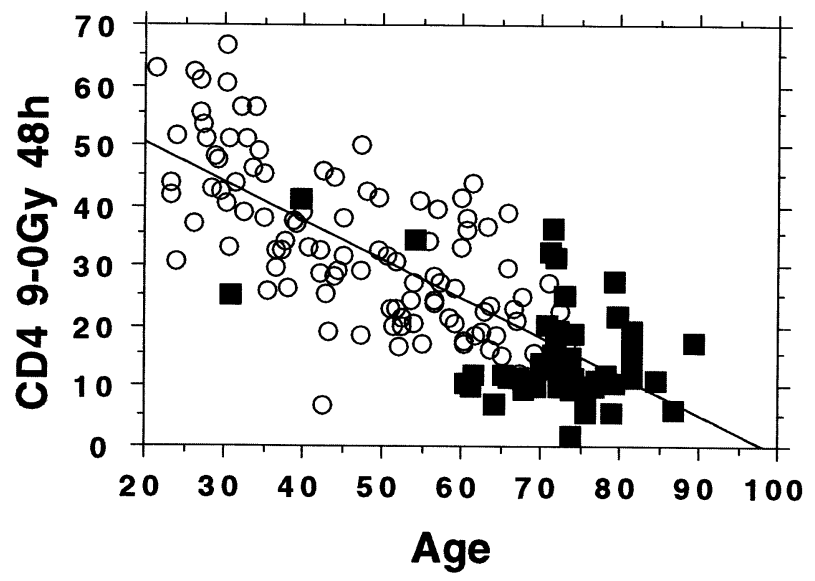

Fig. 3. Percent apoptosis induced by 9-Gy X-rays in CD4 Tlymphocytes as a function of age of blood donor. Open circles = data from a cohort of 105 healthy Red Cross blood donors; filled squares $=$ data from a cohort of 48 non-hypersensitive cancer patients. The background levels (0 Gy) of apoptosis were subtracted. 


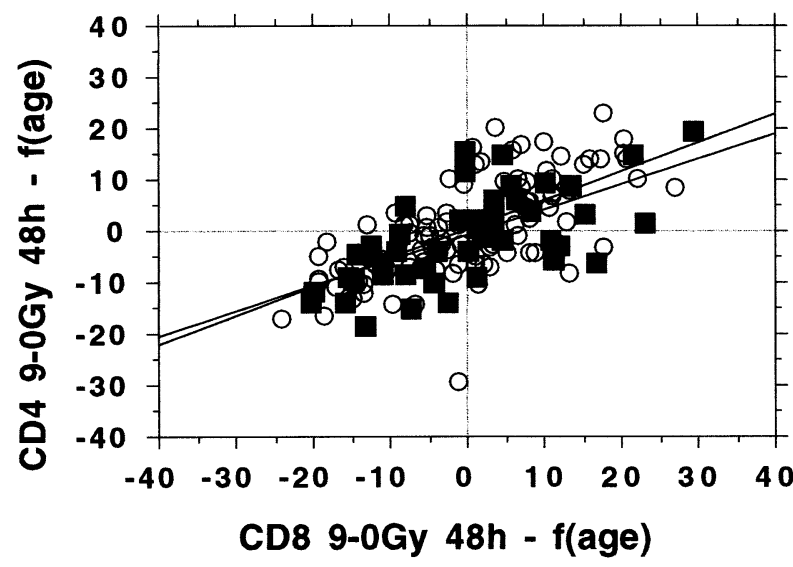

Fig. 4. Age-independent levels (percent) of apoptosis induced by 9-Gy X-rays in the CD8 and CD4 T-lymphocytes. Open circles = data from a cohort of 105 healthy Red Cross blood donors; filled squares $=$ data from a cohort of 48 non-hypersensitive cancer patients. The expected contribution of age, as determined from the regression formula derived from Fig. 3, was subtracted.

older. The regression line has the formula $\mathrm{y}=63.5-$ $0.65 \mathrm{x}, r=0.78$.

Figure 4 compares levels of apoptosis induced by 9-Gy $\mathrm{X}$-rays in the CD4 and CD8 T-lymphocytes after the contribution of age has been accounted for. The expected apoptotic value, based on patient age, was subtracted from the value observed. Data from both the 105 healthy donor cohort and the 48 cancer patient donor cohort were included. The regression lines have the formula $\mathrm{y}=0.56 \mathrm{x}-$ $0.43, r=0.69$; and $\mathrm{y}=0.49 \mathrm{x}-1.06, r=0.65$, for the healthy donors and cancer patient donors, respectively. No significant differences between the two cohorts were observed. Using this combined cohort of 153 normal responders, comparison was made with 21 abnormal responders. The data are presented in Fig. 5. Use of $z$-scores (number of standard deviations from the mean) was made in order to pool data from both 2- and 9-Gy exposures. The response of the CD4 T-lymphocytes is compared with the response of the CD8 T-lymphocytes. Results from three cohorts of donors are displayed; a combined cohort of 153 normal responders, a cohort of 12 hypersensitivity patients ( 6 females, 6 males), and a cohort of 9 ataxia telangiectasia homozygotes.

Figure 6 is a "high-resolution" graph of the hypersensitive cohort response. The apoptotic yield of each patient can be examined in this figure. The $z$-score data were weighted for age; however, less than normal levels of radiationinduced apoptosis were observed in all of these patients. The average CD8-apoptotic $z$-score of these patients is $1.8 \pm 0.66$ standard deviation below the expected mean predicted from the 153 normal responder cohort. To determine levels of significance ( $p$-values) when comparing the normal responder cohort $(n=153)$ with the hypersensitive cohort $(n=12)$, difference-of-means tests were performed. $t$-scores were determined because there were less than 30 individuals in the hypersensitive cohort. Both CD4 and CD8

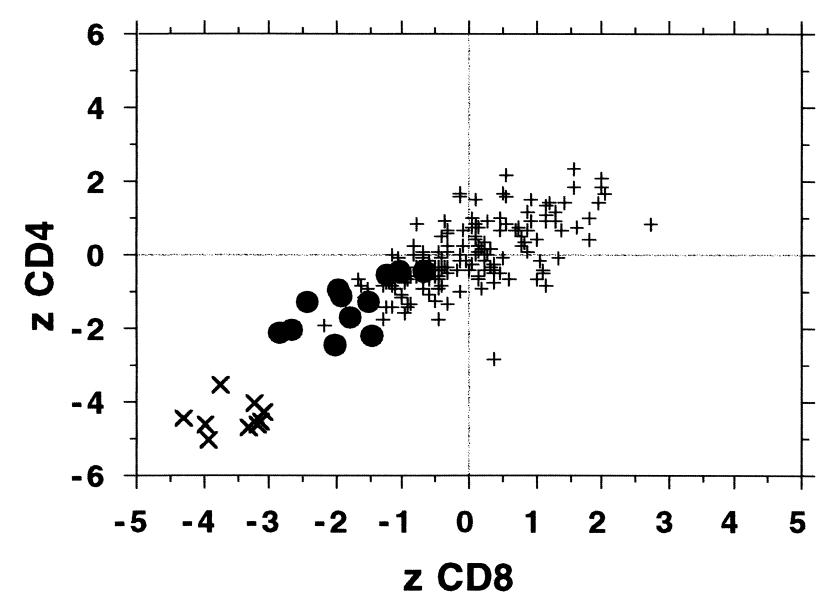

Fig. 5. Apoptotic response of CD8 T-lymphocytes compared with the response of CD4 T-lymphocytes. Results from three cohorts of donors are displayed; + symbols $=$ a combined cohort of 153 $(105+48)$ normal responders; filled circles $=$ a cohort of 12 enhanced radiosensitivity patients; large $\mathrm{X}$ symbols $=$ a cohort of 9 ataxia telangiectasia homozygotes. The expected contribution of age was subtracted and the data are presented as $z$-scores (number of standard deviations from the mean).

T-lymphocyte responses were examined; CD4 T-lymphocytes: $t=4.96, p<0.0001$; CD8 T-lymphocytes: $t=6.71$, $p<0.0001$. The apoptotic response of T-lymphocytes from hypersensitive patients is significantly different to the response of T-lymphocytes from normal individuals.

Table 1 shows the details of the 12 hypersensitive patients and their treatment. The patients are listed in order of increasing apoptotic response based on their average CD4 and CD8 $z$-scores. The age of the hypersensitive patients

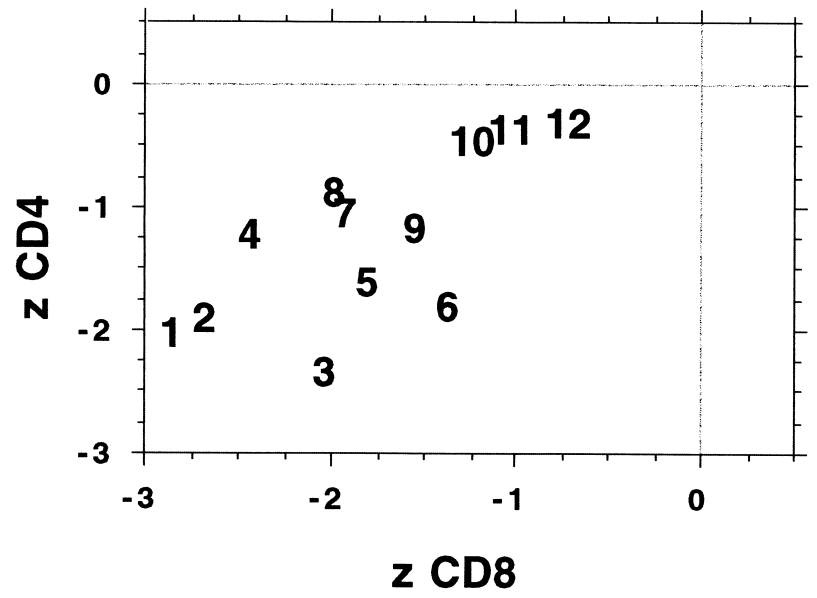

Fig. 6. Apoptotic response of CD8 T-lymphocytes compared with the response of CD4 T-lymphocytes. Results from the cohort of cancer patients who displayed enhanced radiosensitivity. These data are also displayed, at lower resolution, in Fig. 5. The numbered symbols refer to individual patients and are taken from Table 1 . The expected contribution of age was subtracted and the data are presented as $z$-scores (number of standard deviations from the mean). 
Table 1. Hypersensitive patient data

\begin{tabular}{|c|c|c|c|c|c|c|c|c|c|}
\hline Patient & Age & Gender & Diagnosis & Reaction & $\begin{array}{r}\text { Dose } \\
\text { (Gy) }\end{array}$ & $\begin{array}{l}\text { Dose per } \\
\text { fraction } \\
(\mathrm{Gy})\end{array}$ & $\begin{array}{l}\text { Concomitant } \\
\text { chemotherapy }\end{array}$ & $\begin{array}{l}\text { Time until } \\
\text { assay }\end{array}$ & $\begin{array}{l}\text { Avg. } 0 \mathrm{~Gy} \\
\text { apoptosis }\end{array}$ \\
\hline 2 & $51.7 \mathrm{a}$ & Male & Rectal carcinoma* & $\begin{array}{l}\text { Severe late reaction, } \\
\text { ileal occlusion }\end{array}$ & 50.4 & 1.8 & $5-\mathrm{FU}$ & $16.5 \mathrm{mo}$ & 55.18 \\
\hline 5 & $54.2 \mathrm{a}$ & Female & Glioblastoma* & $\begin{array}{l}\text { Brain radionecrosis (late } \\
\text { reaction) }\end{array}$ & 60.0 & 2.0 & None & $12.4 \mathrm{mo}$ & 1.49 \\
\hline 6 & $45.8 \mathrm{a}$ & Female & Rectal carcinoma* & $\begin{array}{l}\text { Severe late reaction, } \\
\text { rectovaginal fistulae }\end{array}$ & 41.6 & $1.6^{\|}$ & None & $5.5 \mathrm{mo}$ & 8.09 \\
\hline 7 & $67.0 \mathrm{a}$ & Male & Lung cancer & $\begin{array}{l}\text { Severe skin \& } \\
\quad \text { esophagitis reactions }\end{array}$ & 36.0 & 2.0 & None & $7.0 \mathrm{mo}$ & 7.25 \\
\hline 10 & $70.9 \mathrm{a}$ & Female & Right breast cancer & $\begin{array}{l}\text { Severe radiation } \\
\text { dermatitis }\end{array}$ & 54.0 & 2.0 & None & $7.0 \mathrm{mo}$ & 3.32 \\
\hline 11 & $54.4 \mathrm{a}$ & Male & Rectal carcinoma* & $\begin{array}{l}\text { Severe skin \& late } \\
\text { enteropathy reactions }\end{array}$ & $45.0^{\#}$ & 1.8 & 5-FU, Mit C & $68.2 \mathrm{mo}$ & 26.20 \\
\hline 12 & $42.2 \mathrm{a}$ & Male & $\begin{array}{l}\text { Adenoid cystic } \\
\text { carcinoma }\end{array}$ & $\begin{array}{l}\text { Severe skin \& mucositis } \\
\text { reactions }\end{array}$ & 55.0 & 2.25 & None & $0.6 \mathrm{mo}$ & 5.03 \\
\hline
\end{tabular}

5-FU = 5-fluorouracil; cis-Pt = cis-platin; Epi = Epirubicin; $\mathrm{CP}=$ cyclophosphamide; Vinbl = vinblastin; Dox $=$ doxorubicin; Mit $\mathrm{C}=$ Mitomycin C.

${ }^{\S}$ Average CD4 and CD8 background (0 Gy) apoptosis (\%).

* Radiotherapy completed.

${ }^{\dagger}$ Required surgery.

* Infarct developed during the third week of postoperative radiotherapy.

" 2 fractions per day.

"I Male breast cancer.

\# 45.0 Gy external therapy and 25.0 Gy brachytherapy, 5 years later the patient received chemotherapy and radiotherapy to treat metastases.

ranged from 41.9 to 75.3 years (average 55 years). The ratio of the sexes was equal. Four breast cancer cases (one male), three rectal cancer cases, and two lung cancer cases are reported. An esophageal cancer case, a head and neck cancer case, and a glioblastoma case are also reported. In no cases were related comorbidities present. In case numbers 1 , $4,7,9,10$, and 12 , very severe early reactions were observed, and therapy was prematurely terminated. In the two cases of severe early reactions where therapy was not stopped, in case number 8 the patient subsequently died, probably related to therapy-associated pulmonary complications (interstitial pneumonitis), and in case number 11, the patient subsequently displayed a severe late reaction: Blindloop syndrome, for several years. Eventually, this patient relapsed and was diagnosed with metastatic mediastinal lymphadenopathies. Treatment of the metastases involved chemotherapy and subsequent radiotherapy, and after 26 Gy, a blood sample was sent for testing. The patient developed severe dysphagia and severe esophagitis. In three other cases (case numbers 2, 5, and 6), severe late reactions also developed. Patient number 2 developed an ileal occlusion and patient number 6 developed rectovaginal fistulae; both required surgery. In case number 5 , a brain radionecrosis was observed following conformal proton therapy. Total radiation doses administered and doses per fraction for all hypersensitive patients are listed in Table 1 .

Concomitant chemotherapy was performed on 6 of the hypersensitive patients. In one case (number 2), chemotherapy extended beyond the end of radiotherapy. In case number 11, the patient received external radiotherapy, brachytherapy, and concomitant chemotherapy. More than 5 years later, when the blood sample was taken, metastases were being treated with chemotherapy (gemcitabine) and subsequent radiotherapy. The background level of apoptosis observed in this case was elevated, presumably as a consequence of the second chemotherapy. Background levels $(0$ Gy) of apoptosis in T-lymphocytes were strongly influenced by chemotherapy. In 6 of 6 patients treated with chemotherapy, the average background level of apoptosis was $24.47 \pm$ $15.77 \%$ (range 13.63-55.18), 5-fold greater than expected. In 6 of 6 patients treated with radiotherapy alone, the average background level of apoptosis was $4.93 \pm 2.45 \%$ (range 1.49-8.09), which is identical to average background levels in the normal responder cohort: $4.95 \pm$ $1.93 \%$. These normal background levels were observed from 0.4 to 12.4 months after completion of radiotherapy, 
and were independent of whether the patients displayed severe early or severe late reactions. These data indicate that treatments based on radiotherapy with concomitant chemotherapy are more noxious, at least to T-lymphocytes, than treatments based on radiotherapy alone.

Although hypersensitive patients who received concomitant chemotherapy tended to display less radiation-induced apoptosis than hypersensitive patients who received radiotherapy alone, a difference-of-means test performed to compare radiation-induced apoptosis revealed that at the $10 \%$ significance level it was not possible to distinguish between the two groups; $t=1.75, p>0.1$. The 5-fold enhanced background levels of apoptosis do not significantly influence subsequent apoptotic response to the radiation doses employed in the leukocyte apoptosis assay. Difference-ofmeans tests were also performed to compare the radiationinduced apoptotic response of these two hypersensitive groups with that of the normal responder cohort. The comparison with patients receiving radiotherapy alone yielded $t=2.71, p<0.0017$ and $t=3.81, p<0.0001$ for CD4 and CD8 T-lymphocytes, respectively; and the comparison with patients receiving concomitant chemotherapy yielded $t=$ 3.70, $p<0.0001$ and $t=5.77, p<0.0001$ for CD4 and CD8 T-lymphocytes, respectively. The radiation-induced apoptotic responses of the CD4 and the CD8 T-lymphocytes from both groups of hypersensitive patients are significantly lower than the responses of the CD4 and the CD8 Tlymphocytes from normal individuals.

\section{DISCUSSION}

Variation is inherent in any biological response assay. It was, therefore, important to determine the reliability of the assay (1). A first estimate of the biological variation of the assay was determined by evaluating the correlation between radiation-induced apoptosis after 2 and 9 Gy exposures. For the CD4 cells, the correlation was highly significant, Pearson's correlation coefficient $r$ was 0.90 . For the CD8 Tlymphocytes this was somewhat lower, $r=0.74$. Statistical analysis using Fischer's transformation of $r$-scores to $z$ scores confirms that the $r$-scores were significantly different even at the $0.01 \%$ level. The reasons for the differences in correlation between the two cell lines is not immediately apparent. It may be that different subsets of CD8 T-lymphocytes display different radiosensitivities and that this contributes to enhanced variation. Comparison of radiationinduced apoptosis after $9 \mathrm{~Gy}$ in CD4 cells with radiationinduced apoptosis after 9 Gy in CD8 cells yields a Pearson's correlation coefficient of 0.77 . There is no significant difference between this value and the one from the CD8 interdose comparison. This indicates that, besides inherent intra-cell-type variation, the information for predicting radiosensitivity gained by analyzing radiation-induced apoptosis in either CD4 or CD8 T-lymphocytes is essentially identical. The slope of the curve in Fig. 2 is less than 1; CD8 T-lymphocytes display on average $25 \%$ more apoptosis than CD4 T-lymphocytes.
Radiation-induced apoptosis was observed to display a significant correlation with age. This correlation is displayed in Fig. 3. The data are from two cohorts of patients from two different cities; a cohort of 105 healthy Red Cross blood donors (Zurich), and a cohort of 48 cancer patient donors (Basel). The data indicate that with each 10 years of life, a dose of 9 Gy X-rays to CD4 T-lymphocytes induces $6.5 \%$ less apoptosis. The older people get, the less able their T-lymphocytes are to mount an apoptotic response to ionizing radiation damage. The lymphocytes were irradiated $e x$ vivo, in blood diluted 10-fold by RPMI medium, which suggests that age-dependent radiosensitivity is an autonomous feature of T-lymphocytes. Effros (22) reported that aging in vivo is associated with an increasing frequency of T-lymphocytes displaying characteristics typical of senescent T cells in culture: shortened telomeres, a loss of CD28 cell surface expression, and an inability to proliferate. She reported that replicative senescence in T-lymphocytes reduces apoptotic response to a variety of stimuli and is associated with increased accumulation of the anti-apoptotic protein Bcl-2. We are investigating whether these findings are the reason for the age-dependent reduction in apoptosis we have observed.

We also examined if the presence of a tumor in a patient interferes with the radioresponsiveness of the T-lymphocytes by comparing the response of the two cohorts (see Fig. 4). The contribution of age, predicted from the regression formula determined from Fig. 3, was subtracted. In the healthy blood donor cohort, after subtraction of the age contribution, Pearson's correlation coefficient was observed to have fallen from 0.77 to 0.69 . This indicates that, although age contributes to $\mathrm{T}$-lymphocyte radiosensitivity, it is not the only factor involved. No significant difference between the healthy donor cohort and the cancer patient cohort was observed. Independent of whether the contribution of age was estimated based on the regression curve of the data from the healthy blood donor cohort alone or from both cohorts together, after the contribution of age had been subtracted, no difference between the two cohorts was observed. The data suggest that the presence of a tumor in a patient does not affect the radiosensitivity of the CD4 or CD8 T-lymphocytes. The presence of a tumor may cause shifts in the relative ratios of leukocyte cell-types, which could result in an apparent change in total lymphocyte response. However, there is no evidence for a systematic release of either pro-apoptotic or anti-apoptotic immunomodulators from the tumors which is capable of altering the radiosensitivity of the T-lymphocyte cell-types.

A clear difference in the sensitivity of CD4 and CD8 T-lymphocytes was observed in individuals who displayed enhanced toxicity to radiation treatment. All of these blood samples displayed less than expected apoptosis. The average CD8 T-lymphocyte apoptotic response of these 12 hypersensitive individuals was $1.8 \sigma$ ( $z$-scores) below the average of the 153 normal responder cohort. Only one individual from the normal responder cohort, but $50 \%$ of the individuals from the hypersensitive cohort, displayed less 
apoptosis than $-1.8 \sigma$. This difference was observed despite the problem of overlapping population distributions associated with this type of study, which tends to reduce the significance of the data. Some individuals who display an apparently hypersensitive reaction will lie at the extremes of the normal responder distribution, where the populations overlap. Testing these individuals for radiation-induced apoptosis will result in a normal response, and their inclusion in the hypersensitive cohort will tend to reduce the numerical significance of the data. It is hoped that an ongoing prospective study will help to define the magnitude of this problem. The reduced apoptosis observed in T-lymphocytes from the hypersensitive individuals supports our hypothesis that such individuals are unable to mount a normal, physiological response to ionizing radiation, and this results in a more severe, pathological reaction (5).

Various causes of differences in radiosensitivity are known, both genetic and epigenetic $(23,24)$. The data demonstrate two immediate sources of differences in apoptotic response which can be classified as epigenetic: cell type and donor age. As a first assumption, the genotypes of an individual's leukocytes are identical. Nevertheless, radiosensitivities of the different cell types are markedly different, and even amongst lymphocytes, differences in radiosensitivities are observed $(4,19,25)$. This emphasizes an important aspect of radiation reactions. Although they are dependent on physical characteristics such as dose, dose rate, and radiation quality, they are also highly dependent on the constellation of cytological radiation response programs available and functioning within cells during the period of damage recognition, repair, and fixation (26). Another source of variation was found to be age of the donor. Because the blood samples were irradiated extracorporally, whatever effect the individuals' body physiology could have which could cause the age dependence, it would have to be made prior to blood accrual. It seems more probable that the age dependence of the cells is related either to a programmed epigenetic effect or to an age-dependent damage accumulation during lymphocyte development in the host. Both of these examples, cell type and donor age, demonstrate how important noninherited factors can be in influencing apoptotic yield in leukocytes.

Two genetic sources of differences in apoptotic yield were also observed, genetic disease and cryptic hypersensitivity. The radiation reaction of patients with ataxia telangiectasia and ICF syndrome (5) both emphasize the role genetic diseases can play. Furthermore, the retrospective study of T-lymphocytes from patients who displayed enhanced toxicity to radiation therapy (cryptic hypersensitivity) clearly demonstrated a compromised apoptotic response specific to these patients. The cohort consistently displayed less radiation-induced apoptosis (on average $-1.8 \sigma$, see Fig. 5) than expected from age-matched controls. Eleven of the hypersensitive responses were more than one standard deviation below the mean. All of the hypersensitive patients had a previous history of radiation exposure, and 6 displayed enhanced levels of background apoptosis associated with concomitant chemotherapy; however, neither of these factors significantly influenced apoptotic response to the radiation doses employed in the leukocyte apoptosis assay.

Apoptosis is believed to protect an organism from the inflammatory effects of necrosis by offering an autonomous death mechanism; a physiologically accommodating and programmed response induced by appropriate and anticipated signals (27). Apoptosis protects from inflammation in a second manner. Apoptotic cells have been demonstrated to induce release of anti-inflammatory cytokines such as interleukin 10 (28). This suggests that enhanced inflammatory reactions to radiotherapy associated with radiosensitivity, as described in this study, arise not only from reduced apoptosis and subsequent enhanced necrosis, but also from confounding effects resulting from the reduced apoptosis, including failure to induce release of anti-inflammatory cytokines. Activation of the complex radiation-induced apoptosis program in nonstimulated peripheral blood lymphocytes is thought to result from initial damage to the DNA and not residual damage $(29,30)$. Such programs consist of three phases: damage recognition, intracellular signaling, and actuation of response (26). During any one of these three phases, modification of the function or the levels of proteins which participate in the induction of apoptosis; such as ATM, p53, Bcl-2, bax, the various caspases, etc., is expected to interfere with the apoptotic yield. Because cellular programs are so complex, until appropriate test methods become available, diagnostic assays should certainly include tests of cellular response with which to substantiate results from protein and gene studies.

There are two important unanswered questions which need to be addressed. The first question concerns correlations with other diagnostic tests of radiosensitivity $(13,31)$. The validity of any diagnostic procedure can only be verified if some "gold standard" exists, in which case results of the procedure are gauged against this (3). Unfortunately, if no such appropriate "gold standard" exists, indirect indicators must be taken. The leukocyte apoptosis assay very clearly demonstrates an age dependence of apoptosis induction. If alternative assays with which this one could be compared do not display a similar age dependence, either they are measuring some other phenomenon or they are simply not as reliable. In either case, their use as a "gold standard" for testing the leukocyte apoptosis assay is questionable. It will, nevertheless, be of interest to compare the leukocyte apoptosis assay with "gold standard" assays of patient radiosensitivity as these become available.

The second question concerns the clinical endpoint of interest. The leukocyte apoptosis assay was developed to predict those patients who would display acute toxicity to radiation therapy. This was a modest goal. It is readily apparent to workers in the field of radiation oncology that late toxicity is the more important parameter limiting dose to the treated volume and the probability of disease-free cure (32). It is intriguing, therefore, that four patients included in the hypersensitive cohort (numbers 2, 5, 6, and 11) 
displayed both significantly reduced levels of apoptosis and severe late effects. Furthermore, all nine ataxia telangiectasia patients displayed significantly reduced levels of apoptosis. We hope that the leukocyte apoptosis assay will provide an indication of the expected magnitude of patientspecific late toxicities. The assay also efficiently demonstrates sensitizing effects of concomitant chemotherapy on the background apoptosis levels in T-lymphocytes. Finally, it is noted that with the increasing number of "dose-escalation" clinical trials, using either conformal radiation therapy or intensity modulated radiation therapy at various sites, the radiation oncologist will welcome a simple and quick normal-tissue radiosensitivity test for use in determining eligibility or stratification criteria of patients to be included in such studies (5). As part of an ongoing prospective clinical trial, these various issues are currently being addressed.

\section{REFERENCES}

1. Crompton NEA, Ozsahin M, Schweizer P, et al. Theory and practice of predictive assays in radiation therapy. Strahlenther Onkol 1997;173:58-67.

2. Meyn AB, Samuel K, Sanderson A, et al. Simultaneous analysis of immunophenotype and apoptosis of murine thymocytes by single laser flow cytometry. Cytometry 1992;13:809821.

3. Daly LE, Bourke GJ, McGilvray J. Interpretation and uses of medical statistics. 4th ed. Oxford: Blackwell Scientific Publishing; 1991.

4. Crompton NEA, Ozsahin M. A versatile and rapid radiosensitivity assay of peripheral blood leukocytes based on DNA and surface marker assessment of cytotoxicity. Radiat Res 1997; 147:55-60.

5. Ozsahin M, Ozsahin H, Shi Y, et al. Rapid assay of intrinsic radiosensitivity based on apoptosis in human CD4 and CD8 T-lymphocytes. Int J Radiat Oncol Biol Phys 1997;38:427440.

6. Lambin P, Marples B, Malaise EP, et al. Hypersensitivity of human tumour cell line to very low radiation doses. Int $J$ Radiat Biol 1993;63:639-650.

7. Iliakis G, Metzger L, Muschel RJ, et al. Induction and repair of DNA double strand breaks on radiation-resistant cells obtained by transformation of primary rat embryo cells with the oncogenes H-ras and v-myc. Cancer Res 1990;50:6575-6579.

8. Ferlini C, Di Cesare S, Rainaldi G, et al. Flow cytometric analysis of the early phases of apoptosis by cellular and nuclear techniques. Cytometry 1996;24:106-115.

9. Milligan CE, Schwartz LM. Programmed cell death during development of animals. Cellular aging and cell death. New York: Wiley-Liss, Inc.; 1996. p. 181-208.

10. Gorman A, McCarthy J, Finucane D, et al. Morphological assessment of apoptosis. In: Cotter TG, Martin SJ, editors. Techniques in apoptosis. London: Portland Press Ltd.; 1994: $1-20$.

11. Marini M, Musiani D, Sestili P, et al. Apoptosis of human lymphocytes in absence or presence of internucleosomal DNA cleavage. Biochem Biophys Res Commun 1996;24:910-915.

12. Trowell OA. The sensitivity of lymphocytes to ionising radiation. J Pathol Bacteriol 1952;64:687-704.

13. Green MHL, Arlett CF, Cole J, et al. Comparative human cellular radiosensitivity: III. $\gamma$-radiation survival of cultured skin fibroblasts and resting $\mathrm{T}$ lymphocytes from the peripheral blood of the same individual. Int J Radiat Biol 1991;59:749765.

14. Radford IR, Murphy TK, Radley JM, et al. Radiation response of mouse lymphoid and myeloid cell lines. Part II. Apoptotic death is shown by all lines examined. Int J Radiat Biol 1994; 65:217-227.

15. Taylor AM, Harnden DG, Arlett CF, et al. Ataxia telangiectasia: A human mutation with abnormal radiation sensitivity. Nature 1975;258:427-429.
16. Meyn MS. Ataxia-telangiectasia and cellular responses to DNA damage. Cancer Res 1995;55:5991-6001.

17. Duchaud E, Ridet A, Stoppa-Lyonnet D, et al. Deregulated apoptosis in ataxia telangiectasia: Association with clinical stigmata and radiosensitivity. Cancer Res 1996;56:1400-1404.

18. Waugh APW, Beare DM, Arlett CF, et al. Comparative of human cellular radiosensitivity: IV. The increased sensitivity of human neonatal cord blood lymphocytes to $\gamma$-irradiation compared with lymphocytes from children and adults. Int $J$ Radiat Biol 1991;59:767-776.

19. Stewart CC, Stevenson AP, Habbersett RC. The effect of low-dose irradiation on unstimulated and PHA-stimulated human lymphocyte subsets. Int J Radiat Biol 1988;53:77-87.

20. Ozsahin H, von Planta M, Müller I, et al. Successful treatment of invasive aspergillosis in chronic granulamatous disease by emergency bone marrow transplantation, G-CSF mobilized granulocytes, and liposomal amphotericin-B. Blood 1998;92: 2719-2724.

21. Menz R, Andres R, Larsson B, et al. Biological dosimetry: The potential use of radiation-induced apoptosis in human T-lymphocytes. Radiat Environ Biophys 1997;36:175-181.

22. Effros RB. Replicative senescence in the immune system: Impact of the Hayflick limit on T-cell function in the elderly. Am J Hum Genet 1998;62:1003-1007.

23. Lewis PD. Variation in individual sensitivity to ionizing radiation. In: Russell Jones R, Southwood R, editors. Radiation and health. Chichester: Wiley; 1987. p. 167-177.

24. Norman A, Kagan AR, Chan SL. The importance of genetics for the optimization of radiation therapy. Am J Clin Oncol 1988;11:84-88.

25. Ayukawa K, Tomooka S, Asano T, et al. Radioresistant CD4CD8 + intrathymic $\mathrm{T}$ cell precursors differentiate into mature CD4+CD8 - and CD4-CD8 + T cells. Thymus 1990;15:6578.

26. Crompton NEA. Programmed cellular response to ionizing radiation damage. Acta Oncol 1998;37:129-142.

27. Wyllie AH, Kerr JFR, Currie AR, et al. Cell death: The significance of apoptosis. Int Rev Cytol 1981;68:251-306.

28. Voll RE, Herrmann M, Roth EA, et al. Immunosuppressive effects of apoptotic cells. Nature 1997;390:350-351.

29. Walter PR, Kohieva L, LeDlanc J, et al. Detection of the initial stage of DNA fragmentation on apoptosis. Biotechniques 1993;15:1032-1040.

30. Vral A, Cornelissen M, Thierens $\mathrm{H}$, et al. Apoptosis induced by fast neutrons versus 60Co gamma-rays in human peripheral blood lymphocytes. Int J Radiat Biol 1998;73:289-295.

31. Kushirio JI, Nakamura N, Kyoizumi S, et al. Absence of correlations between radiosensitivities of human T-lymphocytes in G0 and skin fibroblasts in log phase. Radiat Res 1990; $122: 326-332$.

32. Hall EJ. Radiobiology for the radiologist. 4th ed. Philadelphia: J. B. Lippincott Company; 1994. 